\title{
Model Predictive Torque Vectoring Control for Electric Vehicles Near the Limits of Handling
}

\author{
Efstathios Siampis, Efstathios Velenis and Stefano Longo
}

\begin{abstract}
In this paper we propose a constrained optimal control architecture to stabilize a vehicle near the limit of lateral acceleration using the rear axle electric torque vectoring configuration of an electric vehicle. A nonlinear vehicle and tyre model is employed to find reference steady-state cornering conditions as well as to design a linear Model Predictive Control (MPC) strategy using the rear wheels' slip ratios as input. A Sliding Mode Slip Controller then calculates the necessary motor torques according to the requested wheel slip ratios. After analysing the relative trade-offs between performance and computational effort for the MPC strategy, we validate the controller and compare it against a simpler unconstrained optimal control strategy in a high fidelity simulation environment.
\end{abstract}

\section{INTRODUCTION}

In the past few years it has been recognised that active control of the vehicle's velocity is a very effective strategy in cases of terminal understeer behaviour. This observation is already made by van Zanten et al. [1] who points out that, especially in J-turns where the turning radius is continuously reduced along the trajectory (a scenario typical on highway exits), the ESC's yaw moment correction alone is not sufficient and a controlled velocity reduction is necessary. This remark later resulted in one of the Electronic Stability Control (ESC) new functions, where correction of terminal understeer is achieved by superimposing individual braking of all four wheels on the standard ESC intervention [2]. In [3] a controller providing decoupled longitudinal force and yaw moment inputs at the higher level is combined with a static control allocation scheme to calculate forces and actuator inputs. In [4] a multivariable control architecture to address velocity, yaw and sideslip regulation in terminal understeer is presented. The vehicle model in [4] incorporates nonlinear tyre characteristics and coupling of the longitudinal and lateral tyre forces and a scheduled Linear Quadratic Regulator (LQR) is employed.

In this paper we adopt a multivariable control approach and propose a constrained optimal control architecture in order to address the important in limit handing conditions system constraints. MPC, a control strategy tracing its origins in the chemical processes industry, has been increasingly popular both in academia and industry for its ability to naturally handle multivariable systems constraints. A variety of solutions for active systems using MPC can be found in the literature, ranging from simple online solutions to

E. Siampis, E. Velenis (corresponding author) and Stefano Longo are with the Centre for Automotive Engineering, School of Aerospace, Transport and Manufacturing, Cranfield University, College Road, Cranfield, Bedfordshire, MK43 0AL, e.siampis@cranfield.ac.uk, e.velenisecranfield.ac.uk, s.longo@cranfield.ac.uk complex ones that can be solved only offline. For example, in [5] we find a Linear Time Varying MPC (LTV-MPC) strategy for controlling the lateral dynamics of the vehicle using independent braking of the four wheels. Simulation results show that the LTV-MPC controller can successfully complete the sine and dwell test while respecting the constraints but with a considerable decrease in speed due to the braking strategy used. It is interesting to note here that, although the authors recognise that the problem of overspeeding through a curve is the main reason for failing to follow a reference yaw rate, the proposed controller does not address velocity regulation. Another example of an active lateral dynamics control system using MPC can be found in [6], where a steerby-wire system on a Rear Wheel Drive prototype vehicle is utilized. For the MPC formulation, a bicycle model is used with the front lateral force as input, and bounds are imposed on the yaw rate and sideslip angle in a way similar to the envelope control concept from the aerospace industry. In [7], a hybrid MPC and a switched MPC formulation for a yaw stability controller using Active Front Steering (AFS) and differential wheel braking are presented. Experimental results using the easier to implement switched MPC strategy show that it can successfully stabilize the vehicle in a fast double lane change on a slippery road by enforcing the tyre slip angles constraints. A different approach can be found in [8], where a predictive controller for roadway departure prevention using AFS and braking is presented. The proposed solution utilizes a driver model and future road information to check if the current vehicle state can evolve to a desired future state. For the MPC formulation the cost function penalizes only the control effort, while the road boundaries are set as constraints. Simulation tests show that the controller can successfully keep the vehicle within the lane boundaries in the case of overspeeding through a curve.

In this work we propose a combined velocity, yaw and sideslip regulation strategy for terminal understeer mitigation that accounts for the important in such cases system constraints. We consider a nonlinear vehicle model with nonlinear and coupled tyre forces similar to [4] and design a linear MPC strategy to achieve the reference vehicle behaviour subject to state and input constraints.

\section{Vehicle Model and Reference Generation}

In this section we introduce the vehicle and tyre models used later in this paper. The formulation is similar to the one found in [9], [4], where the interested reader can refer to for more details. 


\section{A. Four-Wheel Vehicle Model}

The Equations Of Motion (EOM) for the four-wheel vehicle model with front wheel steering (Fig. 1) are

$$
\begin{aligned}
m \dot{V} & =\left(f_{F L x}+f_{F R x}\right) \cos (\delta-\beta) \\
& -\left(f_{F L y}+f_{F R y}\right) \sin (\delta-\beta) \\
& +\left(f_{R L x}+f_{R R x}\right) \cos \beta \\
& +\left(f_{R L y}+f_{R R y}\right) \sin \beta \\
\dot{\beta} & =\frac{1}{m V}\left[\left(f_{F L x}+f_{F R x}\right) \sin (\delta-\beta)\right. \\
& +\left(f_{F L y}+f_{F R y}\right) \cos (\delta-\beta) \\
& -\left(f_{R L x}+f_{R R x}\right) \sin \beta \\
& \left.+\left(f_{R L y}+f_{R R y}\right) \cos \beta\right]-\dot{\psi} \\
I_{z} \ddot{\psi} & =\ell_{F}\left[\left(f_{F L y}+f_{F R y}\right) \cos \delta\right. \\
& \left.+\left(f_{F L x}+f_{F R x}\right) \sin \delta\right]-\ell_{R}\left(f_{R L y}+f_{R R y}\right) \\
& +w_{L}\left(f_{F L y} \sin \delta-f_{F L x} \cos \delta-f_{R L x}\right) \\
& +w_{R}\left(f_{F R x} \cos \delta-f_{F R y} \sin \delta+f_{R R x}\right) \\
I_{w} \dot{\omega}_{i j} & =T_{i j}-f_{i j x} r, \quad i=F, R, j=L, R .
\end{aligned}
$$

In the above equations $m$ is the vehicle's mass, $V$ is the vehicle velocity at its Center of Mass (CM), $\beta$ is the sideslip angle at the CM, $\dot{\psi}$ is the yaw rate and $I_{z}$ is the vehicle's moment of inertia about the vertical axis. The moment of inertia of each wheel about its axis of rotation is $I_{w}$, the angular rate of each wheel is $\omega_{i j}$ ( $i=F$ (front), $R$ (rear), $j=L$ (left), $R$ (right)) and the radius of each wheel is $r$. The steering angle on both the front wheels is denoted by $\delta$, and the torque applied on each wheel is $T_{i j}$. The longitudinal and lateral tyre forces are denoted by $f_{i j k}(i=F, R, j=$ $L, R$ and $k=x, y$ ), while the rolling resistances and selfaligning moments at the tyres have been neglected. Finally, $\ell_{F}, \ell_{R}, w_{L}$ and $w_{R}$ determine the location of the center of each wheel with respect to the CM.

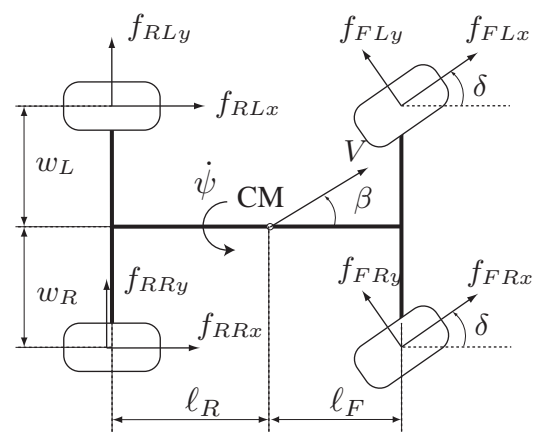

Fig. 1. Four-wheel vehicle model.

\section{B. Tyre Model}

The tyre forces $f_{i j x}$ and $f_{i j y}$ in the above EOM are found as functions of the tyre slip using Pacejka's Magic Formula (MF) [10]. In particular, we obtain the resultant tyre force coefficient as a function of the resultant slip at each tyre from the MF:

$$
\mu_{i j}\left(s_{i j}\right)=\operatorname{MF}\left(s_{i j}\right)=D \sin \left(C \operatorname{atan}\left(B s_{i j}\right)\right),
$$

where $s_{i j}=\sqrt{s_{i j x}^{2}+s_{i j y}^{2}}$ is the resultant tyre slip, with $s_{i j x}$ and $s_{i j y}$ the theoretical longitudinal and lateral slip quantities respectively [10], and $D=\mu_{\max }$ is the tyre/road friction coefficient. Then using the friction circle equations

$$
\mu_{i j x}=-\frac{s_{i j x}}{s_{i j}} \mu_{i j}\left(s_{i j}\right), \quad \mu_{i j y}=-\frac{s_{i j y}}{s_{i j}} \mu_{i j}\left(s_{i j}\right),
$$

we obtain the tyre force coefficients in the longitudinal and lateral direction. Neglecting the pitch and roll rotation along with the vertical motion of the sprung mass of the vehicle, the vertical force $f_{i j z}$ on each of the four wheels can be calculated using the static load distribution and the longitudinal/lateral weight transfers under longitudinal/lateral acceleration [9]. The longitudinal and lateral forces are then given by

$$
f_{i j x}=\mu_{i j x} f_{i j z}, \quad f_{i j y}=\mu_{i j y} f_{i j z} .
$$

\section{Steady-State Cornering Analysis}

In order to derive feasible targets for the controller to follow, steady-state cornering analysis of the four-wheel vehicle model (1) is used. We will neglect for now the wheel speed dynamics and assume actuation of the rear wheels only according to the vehicle topology. Then considering the steering angle and rear wheels longitudinal slip $\left(\delta, s_{R L x}, s_{R R x}\right)$ to be the input of the system (1a)-(1c) and enforcing the steady-state cornering conditions

$$
\dot{V}=0, \quad \dot{\beta}=0, \quad \ddot{\psi}=0,
$$

we can obtain the equilibrium state $\left(V^{\mathrm{ss}}, \beta^{\mathrm{ss}}, R^{\mathrm{ss}}=\right.$ $\left.V^{\mathrm{ss}} / \dot{\psi}^{\mathrm{ss}}\right)$ and input $\left(\delta^{\mathrm{ss}}, s_{R L x}^{\mathrm{ss}}, s_{R R x}^{\mathrm{ss}}\right)$ by providing three of the six unknown variables. In this work we provide the triplet $\left(V^{\mathrm{ss}}, R^{\mathrm{ss}}, \delta^{\mathrm{ss}}\right)$ to complete the calculation. Note that using the calculated longitudinal slip values we can find the steady-state wheel speeds $\omega_{R L}^{\mathrm{ss}}$ and $\omega_{R R}^{\mathrm{ss}}$, and the steady-state torques $T_{R L}^{\mathrm{ss}}$ and $T_{R R}^{\mathrm{ss}}$ through the wheel speed dynamics equation (1d) under steady-state conditions.

Based on the above steady-state analysis, we next examine the feasibility of the vehicle path radius as requested by the driver. Similar to common practice in vehicle stability control [11], we obtain an estimate of the driver's intended path using a neutral steer linear bicycle model under steady-state cornering. The desired path radius is therefore expressed as a function of the driver's steering input by the kinematic relationship $R_{k i n}=\left(\ell_{F}+\ell_{R}\right) / \delta^{\mathrm{ss}}$.

The desired path radius may or may not be feasible depending on the vehicle's velocity. Consider for example the steady-state conditions for a fixed $\delta^{\text {ss }}$ and a range of $V^{\text {ss }}$ in Fig. 2. In all three cases the desired $R^{\mathrm{ss}}=R_{k i n}$ is around $14 \mathrm{~m}$, according to the steering command of $\delta^{\mathrm{ss}}=10 \mathrm{deg}$. Then, for a vehicle velocity of $V^{\mathrm{ss}}=10.75 \mathrm{~m} / \mathrm{s}$ (green curve) the vertical red dashed line corresponding to $R_{k i n}$ intersects the curve of the calculated steady-state conditions, hence the requested $R_{k i n}$ is feasible. On the other hand, if the vehicle velocity is $V^{\mathrm{ss}}=11.25 \mathrm{~m} / \mathrm{s}$ (purple curve) the $R_{k i n}$ is smaller than the minimum achievable $R^{\text {ss }}$ and not longer feasible. In this case the controller will select a steadystate condition such that the desired $R_{k i n}$ becomes feasible 


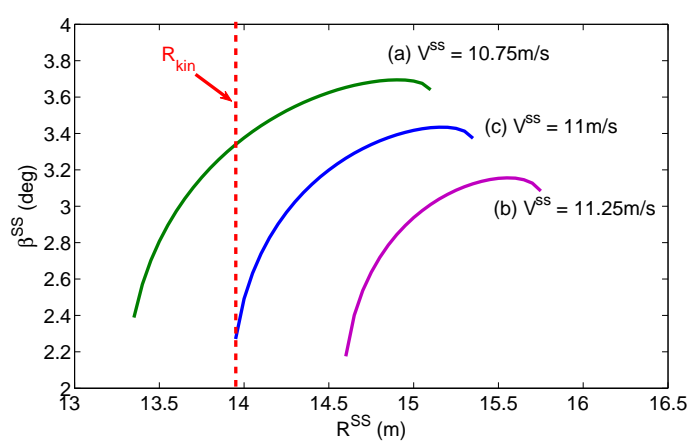

Fig. 2. Selection of target steady-state according to the driver's steering angle command: (a) $R_{k i n}$ feasible at $V^{\mathrm{ss}}=10.75 \mathrm{~m} / \mathrm{s}$; (b) $R_{k i n}$ not feasible at $V^{\mathrm{ss}}=11.25 \mathrm{~m} / \mathrm{s}$; (c) $R_{k i n}$ coincides with the minimum calculated $R^{\mathrm{ss}}$ at $V^{\mathrm{ss}}=11 \mathrm{~m} / \mathrm{s}$.

by reducing the vehicle velocity. Taking into consideration the driver's intention, this velocity reduction will have to be minimal. To this end we select a steady-state velocity such that $R_{k i n}$ coincides with the minimum $R^{\mathrm{ss}}$, which in the above example corresponds to a vehicle velocity of $V^{\mathrm{ss}}=11 \mathrm{~m} / \mathrm{s}$ (blue line).

In summary, the target steady-state condition for the controller to follow is determined using the current steering command from the driver $\left(\delta^{\mathrm{ss}}\right)$, the corresponding kinematic radius $\left(R^{\mathrm{ss}}=R_{k i n}\right)$, and either the current or maximum vehicle velocity $\left(V^{\mathrm{ss}}\right)$ as discussed above.

\section{MPC FORMULATION}

The MPC design is cast in the sampled-data framework by discretizing the continuous-time plant and the associated quadratic cost function. For the linearized continuous-time system about the equilibrium point $\left(x^{\mathrm{ss}}, u^{\mathrm{ss}}\right)$

$$
\dot{x}=A_{c}^{\mathrm{ss}} x+B_{c}^{\mathrm{ss}} u, \quad y=C_{c}^{\mathrm{ss}} x+D_{c}^{\mathrm{ss}} u,
$$

with associated cost function state and input weightings $Q_{c}$ and $R_{c}$ respectively, the discrete-time model using an exact discretization [12] and sampling time $T_{s}$ is

$$
x_{k+1}=A_{d} x_{k}+B_{d} u_{k}, \quad y_{k}=C_{d} x_{k}+D_{d} u_{k} .
$$

Then, assuming $D_{d}=D_{c}=0$ (no feedthrough term) and $C_{d}=I^{n}$ (full state feedback), the MPC regulation problem with horizon $N$ is

$$
\begin{aligned}
\operatorname{minimize} & \sum_{i=0}^{N-1}\left(x_{i}^{T} Q x_{i}+u_{i}^{T} R u_{i}+2 x_{i}^{T} M u_{i}\right) \\
& +x_{N}{ }^{T} P x_{N} \\
\text { subject to } & x_{0}=x_{c u r}, \\
& x_{i+1}=A_{d} x_{i}+B_{d} u_{i}, \quad i=0,1, \ldots, N-1 \\
& u_{i}^{l} \leq u_{i} \leq u_{i}^{h}, \quad i=0,1, \ldots, N-1 \\
& x_{i}^{l} \leq x_{i} \leq x_{i}^{h}, \quad i=1,2, \ldots, N
\end{aligned}
$$

where (2a) is the discretized cost function [12], (2b) sets the initial state $x_{0}$ equal to the current one $x_{\text {cur }},(2 \mathrm{c})$ are the discretized system dynamics and (2d)-(2e) are the state and input inequality constraints respectively. The positive (semi-)definite matrix $Q$ and positive definite matrix $R$ are the weighting matrices on the state error and control effort respectively, and the positive definite matrix $M$ is the crossweighting matrix. The inclusion of the terminal penalty $x_{N}{ }^{T} P x_{N}$ in (2a) ensures closed-loop stability [13], with the matrix $P$ found using the Discrete Algebraic Riccati Equation

$$
\begin{aligned}
P= & \left(B_{d}^{T} P A_{d}+M^{T}\right)^{T}\left(R+B_{d}^{T} P B_{d}\right)^{-1}\left(B_{d}^{T} P A_{d}+\right. \\
& \left.+M^{T}\right)+A_{d}^{T} P A_{d}+Q .
\end{aligned}
$$

Based on the above standard MPC problem, a dense MPC problem using soft constraining on the state [13] is formulated for application in this paper. The necessary $A_{c}, B_{c}, C_{c}$ and $D_{c}$ matrices are updated at each time step according to the current steering command from the driver and the current vehicle velocity, using the analysis of section II-C.

Neglecting the fast wheel speed dynamics, we set $x=$ $\left[\begin{array}{lll}V & \beta & \dot{\psi}\end{array}\right]^{T}$ and $u=\left[\begin{array}{ll}s_{R L x} & s_{R R x}\end{array}\right]^{T}$ for the internal model. Then a Sliding Mode Slip Controller computes the necessary torques on the rear wheels based on the requested longitudinal slips, in a way similar to [14].

1) State Constraints: In order to avoid large yaw rate values, a yaw rate constraint according to the current velocity $V_{\text {cur }}$ is imposed at the beginning of the optimization and fixed throughout the horizon. This constraint is based on the lateral acceleration limit for the current velocity and is coupled to the tyre/road friction coefficient $\mu_{\max }$ [11]:

$$
|\dot{\psi}| \leq \mu_{\max } g / V_{\text {cur }}
$$

Following [5], [15], a constraint on the maximum sideslip angle is also set according to the current velocity:

$|\dot{\beta}|= \begin{cases}2 \frac{k_{1}-k_{2}}{V_{c h}^{3}} V_{c u r}^{3}-3 \frac{k_{1}-k_{2}}{V_{c h}^{2}} V_{c u r}^{2}+k_{1}, & V_{c u r}<V_{c h} \\ k_{2}, & V_{c u r} \geq V_{c h}\end{cases}$ where $V_{c h}$ is the characteristic velocity of the vehicle [16], and $k_{1}$ and $k_{2}$ are chosen at $10 \pi / 180$ and $3 \pi / 180$ respectively. No constraint on the velocity is imposed.

2) Input Constraints: Constraints are also set for the input $u=\left[\begin{array}{ll}s_{R L x} & s_{R R x}\end{array}\right]^{T}$ so that the longitudinal slips on the rear wheels never exceed the maximum allowable slip for safe operation of the vehicle. Using the tyre parameters, as found in Table I, in the simplified MF [10] we find that this limit occurs at around 0.07 so that we set the constraint

$$
\left|s_{R j x}\right| \leq 0.07 \text {. }
$$

3) Torque Constraints: Since we can not directly account for the motor limits in the form of its static torque map (Fig. 3), in the following section we construct an additional constraint on the state and input in order to avoid excessive torque requests from the two motors. Neglecting the wheel speed dynamics, the longitudinal tyre force on each of the rear wheels can be bounded by the maximum and minimum allowable torques

$$
T^{l} \leq f_{R j x} r \leq T^{h},
$$


where $T^{l}$ and $T^{h}$ are calculated as a function of the current wheel speed according to the static torque map at the beginning of each time step and fixed throughout the horizon. Then, linearizing the longitudinal tyre forces $f_{R j x}$ about the equilibrium point, the above double inequality can be expressed in terms of the state $\left[\begin{array}{lll}V & \beta & \dot{\psi}\end{array}\right]^{T}$ and input $\left[\begin{array}{ll}s_{R L x} & s_{R R x}\end{array}\right]^{T}$ and used as an additional constraint in the MPC problem (2).

$$
J_{x}\left[\begin{array}{c}
\widetilde{V} \\
\widetilde{\beta} \\
\dot{\widetilde{\psi}}
\end{array}\right]+J_{u}\left[\begin{array}{c}
\widetilde{s}_{R L x} \\
\widetilde{s}_{R R x}
\end{array}\right] \leq G
$$

where

$$
\begin{gathered}
J_{x}=\left[\begin{array}{lll}
\frac{\vartheta f_{R L x}}{\vartheta V} & \frac{\vartheta f_{R L x}}{\vartheta \beta} & \frac{\vartheta f_{R L x}}{\vartheta \dot{\psi}} \\
\frac{\vartheta f_{R R x}}{\vartheta V} & \frac{\vartheta f_{R R x}}{\vartheta \beta} & \frac{\vartheta f_{R R x}}{\vartheta \dot{\psi}}
\end{array}\right], \\
J_{u}=\left[\begin{array}{ll}
\frac{\vartheta f_{R L x}}{\vartheta s_{R L x}} & \frac{\vartheta f_{R L x}}{\vartheta s_{R R x}} \\
\frac{\vartheta f_{R R x}}{\vartheta s_{R L x}} & \frac{\vartheta f_{R R x}}{\vartheta s_{R R x}}
\end{array}\right], G=\left[\begin{array}{c}
\frac{T_{R L}^{h}}{r}-f_{R L x}^{\mathrm{ss}} \\
\frac{T_{R R}^{h}}{r}-f_{R R x}^{\mathrm{ss}}
\end{array}\right] .
\end{gathered}
$$

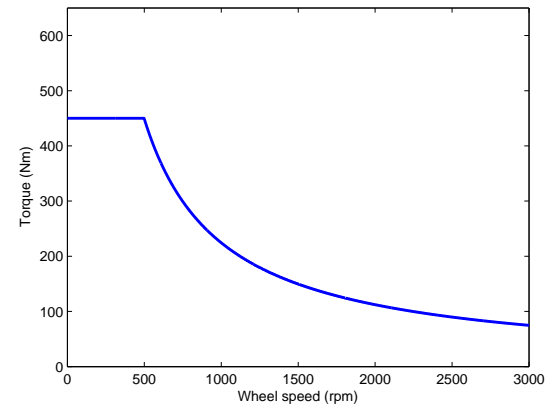

Fig. 3. Motor torque map.

TABLE I

VEHICLE AND TYRE PARAMETERS.

\begin{tabular}{cccc}
\hline Parameter & Value & Parameter & Value \\
\hline$m(\mathrm{~kg})$ & 1420 & $\ell_{F}(\mathrm{~m})$ & 1.01 \\
$I_{z}\left(\mathrm{kgm}^{2}\right)$ & 1027.8 & $\ell_{R}(\mathrm{~m})$ & 1.452 \\
$I_{w}\left(\mathrm{kgm}^{2}\right)$ & 0.6 & $r(\mathrm{~m})$ & 0.3 \\
$w_{L}(\mathrm{~m})$ & 0.81 & $B$ & 24 \\
$w_{R}(\mathrm{~m})$ & 0.81 & $C$ & 1.5 \\
$h(\mathrm{~m})$ & 0.55 & $D$ & 0.9
\end{tabular}

\section{TUNing OF THE MPC StRATEGY}

After choosing the internal model for the MPC problem (2), two are the most important parameters affecting both the performance and computational burden for a MPC formulation: the sampling time $T_{s}$ and the horizon $N$. The effect of varying the sampling time and horizon on the performance and computational effort of the MPC is therefore analysed next. For the evaluation of the performance the closed-loop cost

$$
J_{c l}=\sum_{k}^{\left\lceil\frac{T_{s i m}-T_{s}}{T_{s}}\right\rceil}\left(x_{k}^{T} Q x_{k}+u_{k}^{T} R u_{k}+2 x_{k}^{T} M u_{k}\right),
$$

is used, where $T_{\text {sim }}$ is the chosen simulation time. For the evaluation of the computational effort, the mean time $T_{\text {comp }}$ required from the quadprog solver in MATLAB to construct and solve the MPC problem (2) is used.

\section{A. Impact of Varying the Sampling Time}

First we study the effect of varying the sampling time by comparing the MPC with the unconstrained continuous LQR [4] for a range of sampling times and a horizon equal to the simulation time of $T_{\text {sim }}=10 \mathrm{~s}$. A set of simple simulation scenarios is used, whereas the vehicle is going straight and a step steer input is applied after $2 \mathrm{~s}$. It is assumed at this point that there are no acceleration or braking requests from the driver. For each simulation, the initial vehicle velocity is chosen so that it is $1 \mathrm{~m} / \mathrm{s}$ higher than the maximum velocity allowable for the applied step steering input hence the MPC will regulate the velocity, sideslip angle and yaw rate of the vehicle according to the reference values, as discussed in section II-C. Note that for this set of test scenarios we choose not to constrain the state and input for the MPC, so that we can directly compare it to the LQR as mentioned above.

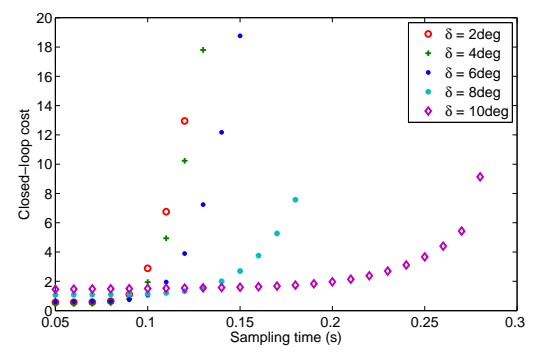

(a) $J_{c l}$ vs $T_{s}$

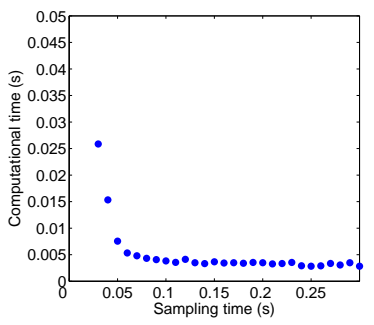

(b) $T_{\text {comp }}$ vs $T_{s}$

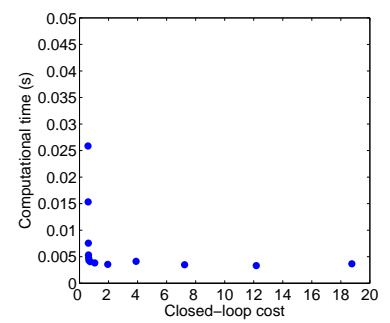

(c) $T_{\text {comp }}$ vs $J_{c l}$
Fig. 4. (a) Closed-loop cost variation with sampling time for a range of step steering inputs $\delta$; (b)-(c) Computational time results and pareto frontier for a step steering input of $\delta=6 \mathrm{deg}$.

Fig. 4(a) shows the variation of the closed-loop cost with sampling time for a range of step steer inputs for the MPC. No considerable changes in performance for sampling times below $0.07 \mathrm{~s}$ can be noticed. Focusing on the simulation results for a step steering input of 6deg, Fig. 4(b)-4(c) show 
the computational time changes with sampling time and the pareto frontier of $T_{c o m p}$ and $J_{c l}$ for this step steering input. We can see that the computational time increases rapidly for sampling times below $0.05 \mathrm{~s}$, so there is a clear tradeoff between closed-loop cost and computational time with changes in the sampling time. This is also evidenced in the pareto frontier of Fig. 4(c), where small reductions in the closed-loop cost below 1 result in large increases in computational time.

\section{B. Impact of Varying the Horizon}

The horizon length of 10 s used in the above section IV-B resulted in long computational times, a large portion of which was spent in constructing the matrices for the dense MPC problem. Using the same test scenario of $6 \mathrm{deg}$ of steering input as above, Fig. 5 shows the increase in construction time with longer horizons. As we can see, the construction time increases exponentially for longer horizons but drops to values as low as $0.005 \mathrm{~s}$ for horizons less than $1 \mathrm{~s}$.

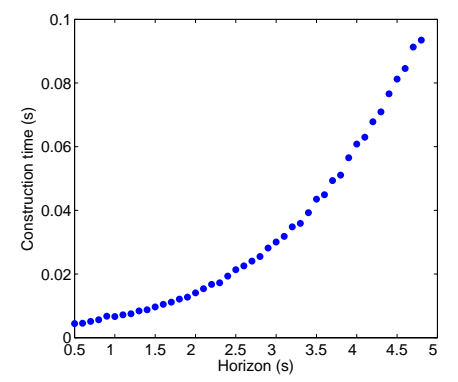

Fig. 5. Construction time needed for a step steering input of $\delta=6 \mathrm{deg}$, a fixed sampling time of $T_{s}=0.05 \mathrm{~s}$ and a range of horizons $N$ from $0.5 \mathrm{~s}$ to $5 \mathrm{~s}$.

\section{Simulation Results}

In the following section we compare the MPC strategy against the LQR and a vehicle without torque vectoring intervention using the high fidelity vehicle model and the driver model available in CarSim. Two are the scenarios under consideration: the first one assesses the understeer mitigation capabilities of the MPC, while the second one assesses its ability to keep the vehicle stable under quick steering input changes. For the MPC we set $T_{s}=0.05 \mathrm{~s}$, a prediction horizon of $N_{p}=1 \mathrm{~s}$ and a control horizon of $N_{u}=0.5 \mathrm{~s}$ according to the analysis of section IV, while we assume again that no acceleration or braking commands come from the driver.

\section{A. U-turn Scenario}

For the U-turn scenario, we use the driver model in CarSim to steer the vehicle through a turn of $56 \mathrm{~m}$ radius. The road is $5.6 \mathrm{~m}$ wide and dry $(\mu=0.9)$, while the entry speed is $85 \mathrm{~km} / \mathrm{h}(23.6 \mathrm{~m} / \mathrm{s})$. In Fig. 6(a) we see the trajectory of the vehicle using the MPC in blue, the vehicle using the LQR in green and the uncontrolled vehicle in red. Both the MPC and the LQR keep the vehicle within the road limits while the uncontrolled vehicle exits the road due to the excessive entry speed.

In Fig. 6(b)-6(e) we see the wheel steering commands from the driver model (assuming a steering ratio of 16:1) along with the velocity, sideslip angle and yaw rate time histories for the three vehicles. Apart from keeping the vehicle within the road limits, the early velocity drop from the MPC and LQR (Fig. 6(c)) allows for smaller wheel steering inputs from the driver model (Fig. 6(b)). In Fig. 6(d) we observe that, while the MPC keeps the sideslip angle values to levels comparable to the ones experienced by the uncontrolled vehicle, the vehicle using the LQR shows much larger values. Finally from Fig. 6(f)-6(g) we observe that the MPC largely keeps the torque requests within the static torque map according to the torque constraint presented in section III-.3.

\section{B. Double-Lane Change Scenario}

For the double-lane change scenario we use again the driver model in CarSim, but this time to follow a predefined path corresponding to a double-lane change manoeuver. The road is assumed to be wet $(\mu=0.4)$ and the entry speed is set to $60 \mathrm{~km} / \mathrm{h}(16.67 \mathrm{~m} / \mathrm{s})$. In Fig. 7 (a) we see the trajectory of the vehicle using the MPC in blue, the vehicle using the LQR in green and the uncontrolled vehicle in red. Both the uncontrolled vehicle and the vehicle with the LQR become unstable towards the end of the manoeuvre and spin out of control.

In Fig. 7(b)-7(c) we see the velocity and sideslip angle time histories for the three vehicles. While the velocity time histories for the MPC and the LQR look similar for the first $5 \mathrm{~s}$, the large sideslip angle values in the case of the LQR result in an unstable condition from which the vehicle cannot recover towards the end of the manoeuvre. In Fig. 7(d)-7(e) we see that the MPC keeps the longitudinal slips within the bound (4). The high frequency oscillations noticed especially in the first half of the simulation for the LQR are the result of a switching strategy used in this case to keep the controller active only within the maximum longitudinal slip threshold [4].

\section{CONCLUSIONS}

In this paper we presented a constrained optimal control architecture to stabilize the vehicle near the limit of lateral acceleration using its rear axle electric torque vectoring configuration. The proposed methodology uses combined velocity, yaw and sideslip regulation to ensure that the reference path, as commanded by the driver, is feasible. Simulations using a high fidelity vehicle model and a driver model in CarSim confirm the effectiveness of the controller in correcting terminal understeer behaviour and the importance of constraining both the state and the input of the system in a fast double-lane manoeuvre.

\section{ACKNOWLEDGMENT}

This work is undertaken within the Evoque_e project, cofunded by the UK's innovation agency, Innovate UK. It is also supported by EPSRC under Grant EP/K502820/1. 


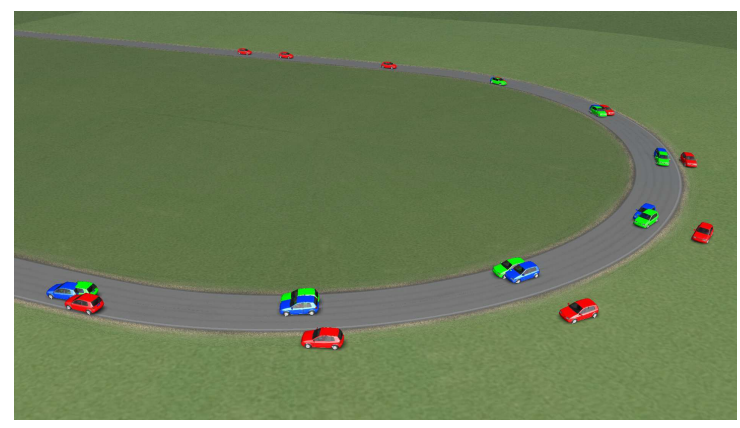

(a) Trajectory

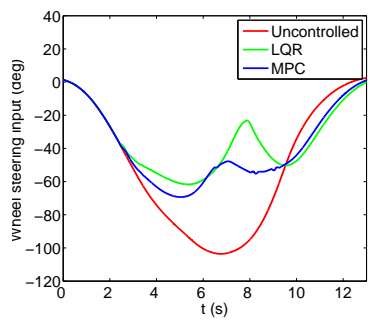

(b) Wheel steering input

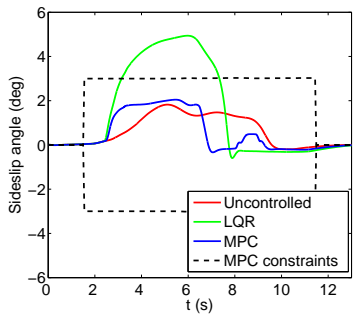

(d) Sideslip angle

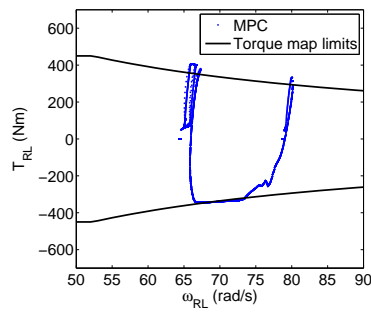

(f) Rear-left wheel torque

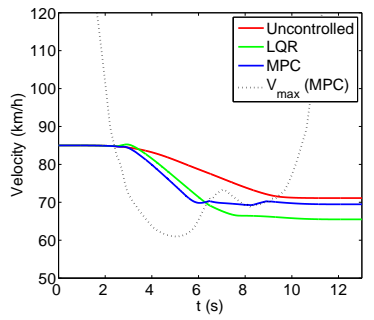

(c) Velocity

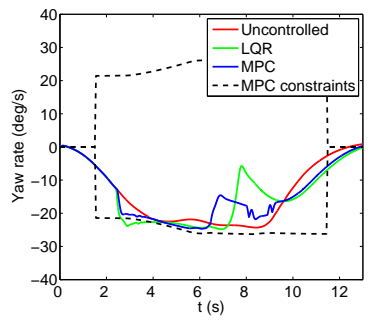

(e) Yaw rate

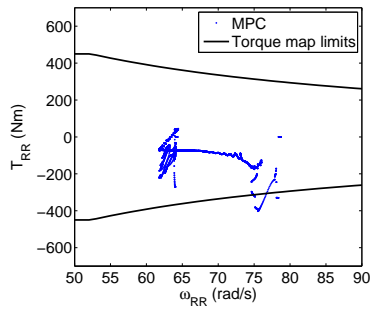

(g) Rear-right wheel torque
Fig. 6. U-turn scenario results (vehicle using the MPC in blue, vehicle using the LQR in green and uncontrolled vehicle in red).

\section{REFERENCES}

[1] A. van Zanten, "Bosch ESP systems: 5 years of experience," 2000, SAE Technical Paper 2000-01-1633.

[2] E. Liebemann and D. Fuehrer, "More safety with vehicle stability control," 2007, SAE Technical Paper 2007-01-2759.

[3] J. Kang, Y. Kyongsu, and H. Heo, "Control allocation based optimal torque vectoring for 4WD electric vehicle," 2012, sAE Technical Paper 2012-01-0246.

[4] E. Siampis, M. Massaro, and E. Velenis, "Electric rear axle torque vectoring for combined yaw stability and velocity control near the limit of handling," in Decision and Control (CDC), 2013 IEEE 52st Annual Conference on, 2013.

[5] O. Barbarisi, G. Palmieri, S. Scala, and L. Glielmo, "LTV-MPC for yaw rate control and side slip control with dynamically constrained differential braking," European Journal of Control, vol. 15, no. 34, pp. $468-479,2009$.

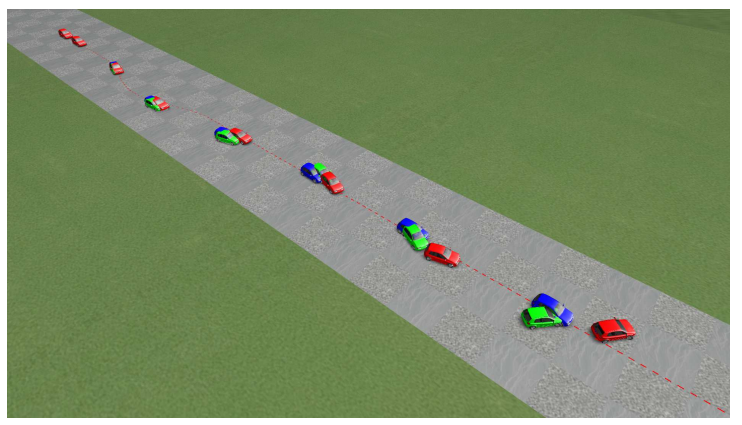

(a) Trajectory

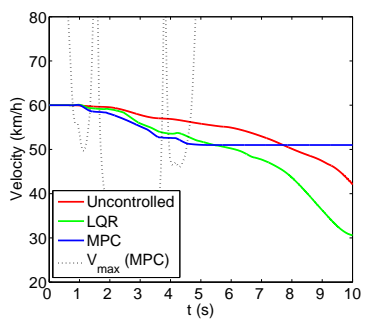

(b) Velocity

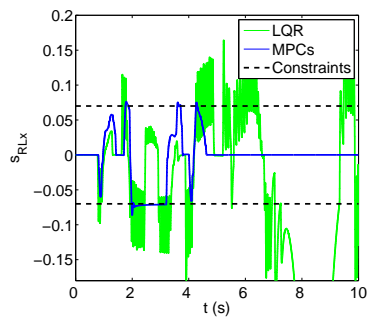

(d) Rear-left wheel long. slip

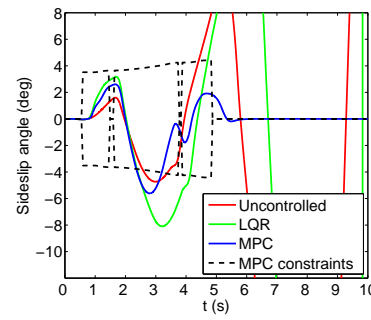

(c) Sideslip angle

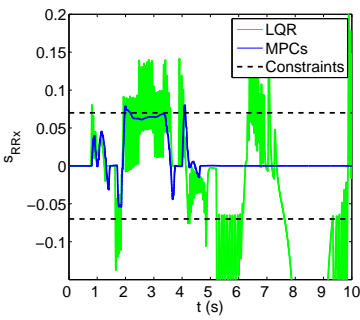

(e) Rear-right wheel long. slip
Fig. 7. Double-lane change scenario results (vehicle using the MPC in blue, vehicle using the LQR in green and uncontrolled vehicle in red)

[6] C. Beal and J. Gerdes, "Model predictive control for vehicle stabilization at the limits of handling," Control Systems Technology, IEEE Transactions on, vol. 21, no. 4, pp. 1258-1269, July 2013.

[7] S. Di Cairano, H. Tseng, D. Bernardini, and A. Bemporad, "Vehicle yaw stability control by coordinated active front steering and differential braking in the tire sideslip angles domain," Control Systems Technology, IEEE Transactions on, vol. 21, no. 4, pp. 1236-1248, July 2013.

[8] A. Gray, M. Ali, Y. Gao, J. Hedrick, and F. Borrelli, "Integrated threat assessment and control design for roadway departure avoidance," in Intelligent Transportation Systems (ITSC), 2012 15th International IEEE Conference on, Sept 2012, pp. 1714-1719.

[9] E. Velenis, D. Katzourakis, E. Frazzoli, P. Tsiotras, and R. Happee, "Steady-state drifting stabilization of RWD vehicles," Control Engineering Practice, vol. 19, no. 11, pp. 1363-1376, November 2011.

[10] E. Bakker, L. Nyborg, and H. Pacejka, "Tyre modelling for use in vehicle dynamics studies," 1987, SAE Technical Paper 870421.

[11] R. Rajamani, Vehicle Dynamics and Control, 2nd ed. Springer, 2012

[12] K. Astrom and B. Wittenmark, Computer-controlled systems, 3rd ed. Prentice-Hall, 1997.

[13] J. Maciejowski, Predictive control with constraints. Prentice Hall, 2002.

[14] E. Velenis, E. Frazzoli, and P. Tsiotras, "On steady-state cornering equilibria for wheeled vehicles with drift," in 48th IEEE Conference on Decision and Control, Shanghai, China, December 16-18 2009.

[15] U. Kienke, Automotive Control Systems. Springer, 2000.

[16] T. Gillespie, Fundamentals of Vehicle Dynamics. Warrendale PA USA: Society of Automotive Engineers SAE International, 1992. 
2015-11-16

\section{Model Predictive torque vectoring control for electric vehicles near the limits of handling}

\section{Siampis, Efstathios}

Institute of Electrical and Electronics Engineers

Efstathios Siampis, Efstathios Velenis and Stefano Longo. Model Predictive torque vectoring control for electric vehicles near the limits of handling. European Control Conference, ECC

2015. 15-17 July 2015, Linz, Austria. pp2553-2558

http://dx.doi.org/10.1109/ECC.2015.7330922

Downloaded from Cranfield Library Services E-Repository 